\title{
Transcriptomics analysis revealed an indirect effect of aqueous cigarette smoke extract in promoting the adhesion of monocytic cells to endothelial cells
}

\author{
Carine Poussin ${ }^{1 *}$, Inka Gallitz², Walter Schlage ${ }^{2}$, Yvonne Steffen$^{1}$, Katrin Stolle², Stephan Lebrun', Julia Hoeng ${ }^{1}$, \\ Michael Lietz ${ }^{2}$
}

From Beyond the Genome 2012

Boston, MA, USA. 27-29 September 2012

\section{Background}

The adhesion of monocytic cells to the 'dysfunctional' endothelium constitutes a critical step in the initiation of atherosclerosis.

Cigarette smoke (CS) has been shown to contribute to the monocyte-endothelial adhesion process. However, the complex underlying molecular mechanisms remain to be unraveled.

\section{Materials and methods}

To investigate the impact of CS on the adhesion of monocytic cells to the endothelium, we developed a conditioned-medium experiment combined with an in vitro adhesion assay intended to mimic the situation found in the systemic compartment. Using a transcriptomics approach followed by confirmation experiments, we were able to identify a key mechanism by which aqueous CS extract in the form of smoke-bubbled phosphate buffered saline (sbPBS) promotes the adhesion of monocytic mono mac 6 (MM6) cells to human umbilical vein endothelial cells (HUVECs).

\section{Results}

While soluble CS constituents elicit a strong oxidative stress response in both cell types, the induced expression of E-selectin, vascular cell adhesion molecule-1 (VCAM-1) and intercellular adhesion molecule-1 (ICAM-1) responsible for the binding of MM6 cells to HUVECs occurs through a pro-inflammatory paracrine effect. Our results show that this effect is largely driven by tumor necrosis factor $\alpha$ (TNF $\alpha)$ produced by MM6 cells exposed to sbPBS.

\section{Conclusions}

Our findings demonstrate that the adhesion of monocytic cells to endothelial cells is promoted through an indirect effect of sbPBS, mainly involving a key soluble factor TNF $\alpha$ and open new avenues for translational research in the comprehension of atherosclerosis development.

\section{Author details}

'Philip Morris International R\&D, Philip Morris Products S.A., Quai Jeanrenaud 5, 2000 Neuchâtel, Switzerland. ²Philip Morris International R\&D, Philip Morris Research Laboratories GmbH, Fuggerstrasse 3, 51149 Cologne, Germany.

Published: 1 October 2012

doi:10.1186/1753-6561-6-S6-P32

Cite this article as: Poussin et al:: Transcriptomics analysis revealed an indirect effect of aqueous cigarette smoke extract in promoting the adhesion of monocytic cells to endothelial cells. BMC Proceedings 2012 6(Suppl 6):P32. 